\title{
Incidence of COVID-19 Infection in Hospital Workers From March 1, 2020 to May 31, 2021 Routinely Tested, Before and After Vaccination With BNT162B2
}

Francesca Larese Filon ( $\sim$ larese@units.it )

University of Trieste

Francesca Rui

University of Trieste

Federico Ronchese

University of Trieste

Paola Michieli

University of Trieste

Corrado Negro

University of Trieste

\section{Research Article}

Keywords: COVID-19, health care workers, incidence, vaccination, BNT162B2

Posted Date: August 18th, 2021

DOI: https://doi.org/10.21203/rs.3.rs-803215/v1

License: (c) This work is licensed under a Creative Commons Attribution 4.0 International License. Read Full License 


\section{Abstract \\ Objective}

To evaluate the incidence of COVID-19 infection in health care workers from the start of COVID-19 pandemic in NE of Italy, to the vaccination with BNT162b2.

\section{Materials and methods}

This was a retrospective cohort study. Health care workers were routinely tested for SARS-CoV-2 infections using real-time polymerase chain reaction tests in nasopharyngeal swabs. Logistic regression was used to calculate incident rate ratios (IRRs) of factors associated to COVID-19.

\section{Results}

A total of 4251 workers were followed-up and an annual incidence of COVID-19 of $13.6 \%$ was found. In March 2021 the incidence of infection was 4.88 and 103.55 cases for 100.000 person-days in vaccinated and nonvaccinated workers, respectively, with an adjusted IRRs of 0.05 (95\% $\mathrm{Cl} 0.02-0.08)$.

\section{Conclusions}

Our study evaluated the monthly incidence in health care workers in Trieste hospitals before and after the vaccination finding the protective effect of BNT162B2 vaccine in $95 \%$ of health care workers routinely tested.

\section{Introduction}

The first index case was identified in March 1, 2020 at Trieste hospital (Italy) in Geriatric ward and since that date hospital's workers were screened for SARS-Cov-2 infection in the first month after contact with cases or in case of symptoms and then [1], on weekly or monthly base, for workers in COVID-19 or non-COVID-19 wards, respectively. On December 27, 2020 a large campaign was launched in Italy to vaccinate its population and health care workers were among the first eligible for vaccination, along with individuals at risk of COVID-19 complication and death [2]. In Friuli Venezia Region hospital's workers started to be vaccinate the same they of the launch and by the end of February, 2021 the $72 \%$ of HCWs in Trieste hospitals received 2 doses of vaccination if never infected by SARSCoV-2 and 1 dose in case of previous infection 3-6 month before.

The BNT162b2 COVID-19 vaccine manufactured by Pfizer and BioNtech demonstrated $95 \%$ efficacy in preventing symptomatic SARS-CoV-2 infection in a phase 3, placebo-controlled randomized clinical trial [3] and it is the first COVID-19 vaccine to receive Emergency Use Authorization by the US Food and Drug Administration [4] and European Medicines Agency [5]. The risk ratio for symptomatic disease, estimated from a cohort of more than 500.000 vaccinated adults and matched unvaccinated controls was 0.06 [6]. However, the association between BNT162b COVID-19 vaccine with asymptomatic infection is crucial and data are scanty [7].

Data on incidence of COVID-19 infection from the start of the pandemic before and after vaccination are limited [7, 8] and none are available for Italy. Previous authors [7, 8] reported a significantly lower incidence of symptomatic 
and asymptomatic SARS-CoV-2 infection after vaccination with an incidence rate ratio adjusted of $0.03(95 \% \mathrm{Cl}$ $0.01-0.06)$ and $0.14(95 \% \mathrm{Cl} 0.07-0.31)$, respectively.

The aim of our study was to evaluate the incidence of COVID-19 infection in hospitals' workers in Trieste (NE of Italy) from the start of pandemic before and after vaccination.

\section{Methods}

This was a retrospective cohort study designed to estimate the incidence of COVID-19 infection in hospital worker's in Trieste public hospitals. The study was conducted in Azienda Sanitaria Universitaria Giuliana Isontina (ASUGI) in Maggiore and Cattinara hospitals, a tertiary medical center that employs approximately 4300 workers. The hospitals have implemented since April 15, 2020 a policy of routinely screening for all workers using nasopharyngeal swabs and PCR-based virus detection. In addition, each case of SARS-CoV-2 infection in health care workers (HCWs) or patients triggered an epidemiological investigation that included the repetition of nasopharyngeal swabs and the collection and recording of symptoms. All hospital workers can receive the vaccination from the first day of the vaccination campaign and throughout the study period on a priority basis.

Data were retrieved from the hospital information system data base, that aggregates data from multiple sources including personnel files, vaccination reports, laboratory data bases, digitized epidemiological questionnaire.

Ethics approval and review were performed according to declaration of Helsinky and were obtained from the Comitato Etico Unico Regionale (CEUR) along with a waiver of written informed consent.

Definition of follow-up period for incidence of COVID-19 in hospitals' workers (phase 1 of the study)

The follow-up started with the first index case identified in Trieste hospitals in March 1, 2020 and continued till May 31, 2021.

The PCR tests were performed according to the screening policy of the hospitals. The screening policy changed during the course of the study as follow: in March only health care workers (HCWs) with respiratory symptoms or in contact with a COVID-cases were tested (period 1), from April 15, 2020 to May 31, 2021 all hospitals' workers including technicians and administrative staff were screened depending of their risk of SARS-CoV-2 exposure (weekly for HCWs in COVID-19 wards and monthly for the others). After the vaccination start (December 27, 2021) routinely screening continued with the same protocol.

All hospitals' workers had open and free access to PCR testing at their own discretion. Hospitals' workers who did not undergo at least one PCR testing during the study period were excluded from the analysis. Workers that contracted SARS-CoV-2 infection during the study contributed to the group of COVID-19 workers while the others were assigned to COVID-19 negative (-) group.

To calculate the incidence of COVID-19 diseases SARS-CoV-2 infected previously were excluded from the analysis. Group assignment for evaluate the efficacy of vaccination (phase II of the study)

Hospitals' workers who received at least 1 vaccine dose between December 27, 2020 and February 28, 2021 were assigned to the vaccinated group. The control group was composed of hospitals' workers who did not receive any doses of the BNT162b2 vaccine during this period. 
To calculate the incidence of COVID-19 diseases in vaccinated and non-vaccinated workers, subjects with previous COVID-19 infection were excluded from the analysis.

The follow-up period for each worker was defining as starting from March 1, 2021 to May 31, 2021. Participants were censored at the first positive PCR test result or in May, 31.

\section{Definitions}

The risk of exposure to COVID-19 subject was classified high for persons working in COVID-19 dedicated wards, medium for persons working in Internal Medicine I and II (where inpatients with respiratory symptoms were hospitalized waiting for a diagnosis), low for workers in other wards and very low for administrative staff and technicians. In case of exposure to various risk situation, the highest risk level of exposure was used.

Participants COVID-19 were defined symptomatic if they had any of the following: temperature greater than $37.5^{\circ} \mathrm{C}$, upper respiratory symptoms (sore throat, cough, and rhinorrhea), lower respiratory symptoms (pneumonia, dyspnea), loss of sense of taste or smell, diarrhea, others (myalgia, malaise, and headache). Symptoms were obtained at the first PCR test and from post-infection epidemiological interviews performed by the Unit of Occupational Medicine involved in infection and prevention control. The participants were considered fully vaccinated more than 7 days after the second dose of the vaccine.

\section{Study outcomes}

The primary outcome was the incidence of SARS-CoV-2 infection in hospitals' workers from the start of the pandemic to February 28, 2021. The secondary outcome was the incidence of SARS-CoV-2 infection from March 1 to May 31, 2021 in vaccinated and non-vaccinated workers. Outcomes were analyzed for pre-defined subgroups according to age, sex, work task, wards according to the estimated level of exposure.

\section{Statistical analysis}

Continuous variables are expressed as mean and standard deviation (SD) for normally distributed variables and as median (interquartile range) for non-normally distribute variables. Categorical variables are expressed as number (percentage) of hospital workers within each group The groups were compared using $t$ test for normally distributed continuous variables and chi-square tests for categorical variables. Missing values for categorical variables were considered as separate category. A significance for $p<0.05$ two sided was considered significant.

\section{Incidence rate analysis}

The incidence rates were defined as the number of positive SARS-CoV-2 cases divided by number of person-days during the surveillance time.

The adjusted incidence rate ratio (IRR) was estimated using a multivariate Poisson regression model with confirmed cases as a response variables and group assignment (COVID-19 positive and negative for study phase I and vaccinated and, non-vaccinated for study phase II), age sex work tasks, wards and number of PCR tests for each health care workers in the time frame under observation. The IRRs were computed by exponentiating the group assignment coefficient from the final regression model and $95 \% \mathrm{Cl}$ s and $\mathrm{P}$ values were estimated from the model. 
All statistical analysis was performed using STATA (StataCorp LLC, College Station, Texas USA).

\section{Results}

Phase I study. Incidence of COVID-19 acquired infection from March 1, 2020 to February 28, 2021

One-year incidence study was performed on the cohort of HCWs at Trieste Hospitals (Maggiore and Cattinara) constituted of 4300 workers (Fig. 1). Forty-nine workers were excluded for incomplete data, while 4231 workers were followed up from the start of the SARS-CoV-2 pandemic in Trieste (Italy) for 12 months. During this period 46,274 PCR test was performed in nasopharyngeal swabs of HCWs that were routinely screened to detect early COVID-19 infection. At December 27, 2020 started the vaccination campaign in Italy for HCWs and before February 20, 2021, 72\% of the workforce get two-vaccination doses. During the 12 months period 578 HCWs acquired COVID-19 (13.6\%). The Table 1 reported the characteristics of the cohort followed. Sex distribution was similar for infected and non-infected workers, but COVID-19 HCWs resulted significant younger (44.7 \pm 11.1 years vs $47.2 \pm 10.6$ years, $p<0.001$, respectively) and subjects with less than 31 years resulted more affected ( $p<$ $0.001)$. The majority of workers that acquired the infection was nurses $(45.2 \%)$ and nurse aids (21.8\%). Prevalence of infection resulted different in relationship to exposure risk: in some ward involved in treatment of COVID-19 patients, infected workers were more than $50 \%$ (Long-term Covid) or less beyond (Geriatric and Infective diseases with $46.5 \%$ and $47.9 \%$ of the workforce that resulted infected). In other divisions such as Rehabilitation and Pulmonology, both COVID-19 wards, the $21.1 \%$ and $30.5 \%$ of HCWs acquired the infection. Lower percentages of COVID-19 were found in other wards not involved in treatment of COVID-19 patients such as surgeries (10.5\%), other medicine wards (7.3\%). To note that HCWs in Anesthesia and Resuscitation, involved in treatment of COVID19 patients, presented very low prevalence of SARS-CoV-2 infection (8.6\%). On the overall cohort, each worker

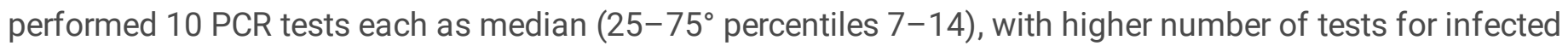
HCWs that repeated PCR more often (median 14 tests, $25-25^{\circ}$ percentiles $10-19$ ), $\mathrm{p}=0.03$.

New cases of SARS-CoV-2 infection during one year are reported in Fig. 2, were is clearly shown the two waves of infection that characterized Italy, with the first wave from March to May, 2020 and the second wave from October, 2020 to February, 2021. The start of vaccination in December 27, 2020 caused a deep decline of cases until 0 at the mid of February, 2021.

The $52.1 \%$ of subjects reported symptoms the day of the first positive PCR, while the others were asymptomatic. During the follow-up the $16.4 \%$ of workers remained asymptomatic while the others developed symptoms, mainly mild at upper respiratory airways (sore throat, cough, rhinorrhea: $54.5 \%)$ fever $>37.5^{\circ} \mathrm{C}(47.2 \%)$ and loss of taste and smell (27.8\%). Thirty-three workers had lower respiratory symptoms (5.7\%) and one died.

Monthly incidence of SARS-CoV-2 infection is reported in Table 2 as person-days: in March, 2020 the incidence was 72.69 cases per 100.000 person-days declining in April and May, 2020. No cases were recorded in June and July, 2020 while few cases occurred in August and September, 2020. The second wave of infection started in October, 2020 with an incidence of 55.7 cases per 100.000 person-days, that increased to 97.57 and 93.97 cases per 100.000 person-days in November and December, 2020, respectively. In January the incidence was still high (61.12 cases per 100.000 person-days) and finally declined in February, 2021 (13.56 cases per 100.000 persondays). 
Table 3 reported the incidence risk ratio (IRR) and 95\% confidence intervals (CI) of factors associated with SARSCoV-2 infection in HCWs calculated using the Poisson regression analysis. Younger age was confirmed as a factor involved in COVID-19 acquired infection together with work in some COVID-19 dedicated wards. The highest IRR was found for HCWs in Long-term Covid (IRR 7.15, 95\% Cl 4.6-11.1) compared to other medicine wards. To note that Anesthesia and Resuscitation, a COVID-19 ward did not presented an increased risk of infection.

Phase II study

Incidence of COVID-19 acquired infection from March 1, 2021 to May 31, 2021

Since March 1, 2021 the 72\% of workers resulted fully vaccinated against COVID-19 with the second shot done before the mid of February 20, 2021. In Table 4 incidence of SARS-CoV-2 infection was compared in vaccinated and non-vaccinated workers. Only 6 vaccinated HCWs acquired the infection in the 3 months considered, while the incidence resulted very high in March for non-vaccinated people (103.55 cases per 100.000 person-days, declining in April (50.25 cases per 100.000 person-days) and May (13 cases per 100.000 person-days) in correspondence of the declining of the infection in general population in Italy. The Incidence rate ratio between vaccinated and nonvaccinated workers resulted 0.05 (95\% Cl 0.02-0.08) in March and April 2021.

\section{Discussion}

Our study investigate the incidence of COVID-19 acquired infection in HCWs regularly screening with PCR detection of SARS-CoV-2 in nasopharyngeal swabs in NE of Italy. Data on incidence of COVID-19 infection are very limited in this long timeframe, with a regular screening to identify asymptomatic or pre-symptomatic infection in HCWs, with the aim to reduce the transmission to colleagues and patients.

In France, the incidence rate was estimated to be 5\% [9] between March 17 and April 20, 2020), in Italy Colaneri et al. [10] reported an incidence of 11.33\% (9.72-13.21) from February 22 to May 8, 2020, in Boston Lan et al. [11] reported higher incidence (14\%) between March 9 and April 15, but all authors tested only symptomatic workers. A review of literature, estimated a pooled prevalence of $11 \%$, higher for symptomatic workers (19\%) and lower for asymptomatic (5\%) [12].

Asymptomatically infected individuals are estimated to account for $40-45 \%$ of SARS-CoV-2 infections and may spread the virus per extended periods [13]. Asymptomatic infections have been proposed as the major barrier to controlling the spread of SARS-CoV-2 infection and are a possible explanation for the rapid evolution of the COVID-19 pandemic [13].

In our cohort the $47.9 \%$ of subjects were asymptomatic the day of the first PCR positive, then during the follow-up of symptoms the $16.4 \%$ remained asymptomatic while the others developed symptoms, mainly on the upper airways. For that reason, the regular screening of workers using the PCR SARS-CoV-2 detection in nasopharyngeal swabs was the first line (before vaccination) to control the spread of infection in hospital workers.

In our study, the incidence of COVID-19 infection was 13.6\% between March 1, 2020 and February 28, 2021: Colaneri et al. reported a cumulative incidence of $11.33 \%$ in another Italian hospital analyzing infections from February 22 to May 8, 2020. The risk to be COVID-19 positive decreased with age (IRR 0.99; 95\% CI0.98-0.99) and people with less than 31 years resulted more infected compared to older workers. Studies performed testing only symptomatic workers did not find an increased risk in young people $[9,10]$ probably because they did not test 
routinely HCWs, loosing younger subjects that had frequently asymptomatic infection. Similar risk to acquire the infection was fund in different job categories with the exception of workers without direct contact with patients ("Others" IRR 0.70; 95\% Cl 0.50-0.98). Big differences were found in wards in which the highest risk was found in some COVID-19 dedicated wards (Long-term, Infective diseases, Geriatrics) and where occurred some infection clusters (Emergency medicine, Internal medicine), while the risk to acquire the infection was lower in "Other medicines", in Surgeries, in Anesthesia and Resuscitation. This finding is similar to other reports in which COVIDwards presented a higher risk of infection for employed workers $[9,10]$. To evaluate the trend of infection during one year, we calculated the incidence per 100.000 person-days finding a wide variation, as happened in regional general population in Italy. The pandemic started at March 2020 with 72.60 (95\% Cl 64-98) cases per 100.000 person-days and decreased until 0 in June and July, after the Italian lock down. The second wave of the pandemic started slowly at the end of the summer and reached the peak in November and December with 97.57 (95\% Cl 9399) and 93.97 (95\% Cl 88-98) cases per 100.000 person-days. Vaccination for all HCWs was available since December 27, 2020, the decrease in COVID-19 infections started in February with 0 cases from the second half of the month. The incidence in the last 3 months considered was calculated considering vaccinated and nonvaccinated HCWs showing an extremely low number of cases in vaccinated people and the highest incidence in March, 2021 for non-vaccinated HCWs with an incidence of 103.55 cases per 100.000 person-days. The IRR between vaccinated and non-vaccinated was 0.05 (95\% $\mathrm{Cl} 0.01-0.08)$ in March and April 2021. Our results are similar to those reported by Angel in 2021 [7] in Israel with an incidence rate of 4.5 for 100.000 person-days in fully vaccinated HCWs, while they found higher incidence in non-vaccinated people (148.8 cases for 100.000 persondays). Tang et al. [8] reported an incidence rate ratio for confirmed COVID-19 cases per person-days in vaccinated compared to unvaccinated group of 0.04 (95\% $\mathrm{Cl} 0.02-0.09)$.

Our study found an IRR of 0.05 for vaccinated compared to non-vaccinated HCWs, corresponding to an estimated vaccine effectiveness of (1-IRR) 95\%, that is the same reported in the phase 3 randomized clinical trial [3] and the risk ratio of 0.06 observed in a study of the nationwide vaccination in Israel [6]. Benenson et al. [14] found a very low incidence of COVID-19 in vaccinated workers compared to non-vaccinated. The weekly incidence of Covid-19 since the first dose declined notably after the second week; the incidence of infection continued to decrease dramatically and then remained low after the fourth week. Amit et al.[15] reported a rate of reduction of $85 \%$ in vaccinated vs non-vaccinated HCws.

Vaccination permitted to reduce COVID-19 infection in HCWs and vaccine's coverage of $72 \%$ of HCWs in our hospitals permitted to reduce the acquired infection also in non-vaccinated HCWs in May, 2021.

Our study highlighted COVID-19 infection in HCWs in Italy from the start of the pandemic in our region (March 1, 2020) for one year and then it evaluated COVID-19 incidence of infection in fully vaccinated and non-vaccinated workers. On the best of our knowledge, our study in the first in Italy and one of the few in the world $[16,17]$ that evaluate the monthly incidence of COVID-19 infection from the start of the pandemic till now, using a weekly or monthly PCR screening for all HCWs according with their risk to be exposed to COVID-19 infected persons. The screening permitted to identify positive asymptomatic or pre-symptomatic subjects that need to be restricted from work to avoid the spread of infection to patients and colleagues. The effectiveness of periodical screening is demonstrated also by the lower incidence of COVID-19 acquired infection in non-vaccinated people compared to others available data: i.e. Angel reported an incidence in non-vaccinated HCWs of 149.8 cases for 100.000 personday, that is higher than our data for March, 2021 (103.55 cases for 100.000 person-day), although also these authors performed the periodical screening in HCWs during the surveillance period. 
Our study described the monthly incidence of COVID-19 acquired infection during the first year of COVID-19 pandemic in HCWs that were periodically screened to detect SARS-Cov-2 in nasopharyngeal swabs using RT-PCR technique. Moreover, we demonstrated the effect of vaccination with BNT16b2 showing a sharp decrease of cases in fully vaccinated individuals. Risk factors such as young age and workplaces were identified as significantly related to COVID-19 acquired infection.

The strength of our study is the long follow-up that permitted to define a monthly incidence of COVID-19 infection in our HCWs before and after vaccination. The routinely PCR detection in nasopharyngeal swab permitted to identify asymptomatic and pre-symptomatic infection, to limit the spread of the infection to patients and other workers [18-19].

Limitations of the study

This study has several limitations. First it is a single-center, retrospective cohort study, thou our findings might be not generalized. Second, the effect of vaccination was studied for three months in witch progressively decreased COVID-19 infection in our region. Third, other confounders may be present there were unaccounted for in the regression analysis, for example health status and relationship between health status and vaccination.

\section{Conclusions}

Our study reported data on the incidence of COVID-19 infection in a cohort of HCWs regularly screened with PCR from April, 2020 in Friuli Venezia Giulia Region (NE of Italy) till the end of May, 2021. Among HCWs, vaccination with BNT162b2, was associated to a sharp decline of incidence of COVID-19 infection with an IRR of 0.05 in vaccinate compared to non-vaccinate workers. We confirmed the $95 \%$ efficacy of this vaccination for the prevention of SARS-CoV-2 infection.

\section{Declarations}

\section{Author Contributions}

Larese Filon $\mathrm{F}$ and Negro $\mathrm{C}$ had full access to all data in the study and take responsibility for the accuracy of data analysis. Concept and design: Larese Filon F and Negro C. Acquisition, analysis and interpretation of data: Larese Filon F, Negro C, Ronchese F. Drafting of the manuscript: Larese Filon F, Rui F, De Michieli P. Critical revision: all authors. Statistical analysis: Larese Filon F.

\section{Conflict of interest Disclosures}

All Authors declare they have no conflict of interest

\section{Funding source}

None

\section{Competing interests}

None declared.

\section{Data availability statement}


Data are available upon reasonable request.

\section{References}

1. Piapan L, De Michieli P, Ronchese F, Rui F, Mauro M, Peresson M, Segat L, D'Agaro P, Negro C, Bovenzi M, Larese Filon F. COVID-19 outbreak in healthcare workers in hospitals in Trieste, North-east Italy. J Hosp Infect. 2020 Nov;106(3):626-628. doi: 10.1016/j.jhin.2020.08.012

2. Istituto Superiore di Sanità (ISS). Speciale COVID-19 vaccini https://www.iss.it/vaccini-covid-19 (accessed February 2, 2021)

3. Polack FP, Thomas SJ, Kitchin N, et al; C4591001 Clinical Trial Group. Safety and efficacy of the BNT162b2 mRNA Covid-19 vaccine. N Engl J Med. 2020;383(27):2603-2615. doi:10.1056/NEJMoa2034577

4. US Food and Drug Agency. Emergency Use Authorization for Pfizer-BioNTech COVID-19 vaccine. Accessed February 25, 2021. https://www.fda.gov/emergency-preparedness-and-response/coronavirus-disease-2019covid-19/pfizer-biontech-covid-19-vaccine

5. European Medicines Agency. EMA recommends first COVID-19 vaccine for authorisation in the EU. https://www.ema.europa.eu/en/news/ema-recommends-first-covid-19-vaccine-authorisation-eu (accessed February 8, 2021)

6. Dagan N, Barda N, Kepten E, et al. BNT162b2 mRNA Covid-19 vaccine in a nationwide mass vaccination setting. N Engl J Med. 2021;384(15): 1412-1423. doi:10.1056/NEJMoa2101765

7. Angel Y, Spitzer A, Henig O, Saiag E, Sprecher E, Padova H, Ben-Ami R. Association Between Vaccination With BNT162b2 and Incidence of Symptomatic and Asymptomatic SARS-CoV-2 Infections Among Health Care Workers. JAMA. 2021 May 6. doi: 10.1001/jama.2021.7152. Online ahead of print.

8. Tang L, Hijano DR, Gaur AH, Geiger TL, Neufeld EJ, Hoffman JM, Hayden RT. Asymptomatic and Symptomatic SARS-CoV-2 Infections After BNT162b2 Vaccination in a Routinely Screened Workforce. JAMA. 2021 May 6. doi: 10.1001/jama.2021.6564

9. Krastinova E, Garrait V, Lecam MT, Coste A, Varon E, Delacroix I, Si Ali A, Jung C, Smati M, Cherbit M, Maître B, Pairon JC, Andujar P. Household transmission and incidence of positive SARS-CoV-2 RT-PCR in symptomatic healthcare workers, clinical course and outcome: a French hospital experience. Occup Environ Med. 2020 Dec 4:oemed-2020-106866. doi: 10.1136/oemed-2020-106866

10. Colaneri M, Novelli V, Cutti S, Muzzi A, Resani G, Monti MC, Rona C, Grugnetti AM, Rettani M, Rovida F, Zuccaro V, Triarico A, Marena C. The experience of the health care workers of a severely hit SARS-CoV-2 referral Hospital in Italy: incidence, clinical course and modifiable risk factors for COVID-19 infection. J Public Health (Oxf). 2021 Apr 12;43(1):26-34. doi: 10.1093/pubmed/fdaa195.

11. Lan FY, Filler R, Mathew S, Buley J, Iliaki E, Bruno-Murtha LA, Osgood R, Christophi CA, Fernandez-Montero A, Kales SN. COVID-19 symptoms predictive of healthcare workers' SARS-CoV-2 PCR results. PLoS One. 2020 Jun 26;15(6):e0235460. doi: 10.1371/journal.pone.0235460. eCollection 2020.

12. Gómez-Ochoa SA, Franco OH, Rojas LZ, Raguindin PF, Roa-Díaz ZM, Wyssmann BM, Guevara SLR, Echeverría LE, Glisic M, Muka T. COVID-19 in Health-Care Workers: A Living Systematic Review and Meta-Analysis of Prevalence, Risk Factors, Clinical Characteristics, and Outcomes. Am J Epidemiol. 2021 Jan 4;190(1):161-175. doi: 10.1093/aje/kwaa191. 
13. Oran DP, Topol EJ. Prevalence of asymptomatic SARS-CoV-2 infection: a narrative review. Ann Intern Med. 2020;173(5):362-367. doi:10.7326/M20-3012

14. Benenson S, Oster Y, Cohen MJ, Nir-Paz R. BNT162b2 mRNA Covid-19 vaccine effectiveness among health care workers. N Engl J Med. Published online March 23, 2021. doi:10.1056/NEJMc2101951

15. Amit S, Regev-Yochay G, Afek A, Kreiss Y, Leshem E. Early rate reductions of SARS-CoV-2 infection and COVID19 in BNT162b2 vaccine recipients. 2021;397(10277):875-877. doi:10.1016/S0140-6736(21)00448-7

16. McNicholas JE, Kosnik R, Blanc PD, Taylor BR, Guntur S. Temporal Trends in COVID-19 Incidence in Two Healthcare Worker Cohorts. J Occup Environ Med. 2021 Jun 1;63(6):528-531. doi: 10.1097/JOM.0000000000002208.

17. Suárez-García I, Martínez de Aramayona López MJ, Sáez Vicente A, Lobo Abascal P. SARS-CoV-2 infection among healthcare workers in a hospital in Madrid, Spain. J Hosp Infect. 2020 Oct;106(2):357-363. doi: 10.1016/j.jhin.2020.07.020. Epub 2020 Jul 21.

18. Lee $\mathrm{S}, \mathrm{Kim} \mathrm{T}$, Lee $\mathrm{E}$, et al. Clinical course and molecular viral shedding among asymptomatic and symptomatic patients with SARS-CoV-2 Infection in a community treatment center in the Republic of Korea. JAMA Intern Med. 2020;180(11):1447-1452. doi:10.1001/jamainternmed.2020.3862

19. Nogrady B. What the data say about asymptomatic COVID infections. 2020;587(7835):534-535. doi:10.1038/d41586-020-03141-3

\section{Tables}

Table 1 Characteristics of the population studied and acquired COVID-19 infection from March 1, 2020 to February 28,2021 


\begin{tabular}{|c|c|c|c|c|}
\hline & Covid-19+ & Covid 19 - & Total & $\mathrm{P}$ \\
\hline Total n. (\%) & 578 (13.6\%) & $3673(86.4 \%)$ & $4.251(100 \%)$ & \\
\hline Women n. (\%) & 394 (68.2\%) & $2550(69.4 \%)$ & $2.944(69.3 \%)$ & 0.542 \\
\hline Age mean (SD) & $44.7 \pm 11.1$ & $47.2 \pm 10.6$ & $46.9 \pm 10.7$ & 0.000 \\
\hline \multicolumn{5}{|l|}{ Age classes n. (\%) } \\
\hline$-\quad<31$ & $87(15.1 \%)$ & $335(9.1 \%)$ & $422(9.9 \%)$ & \multirow{5}{*}{0.000} \\
\hline $\begin{array}{l}-\quad 31-40\end{array}$ & 115 (19.9\%) & $660(18.0 \%)$ & 775 (18.2\%) & \\
\hline$-\quad 41-50$ & 170 (29.4\%) & $1061(28.9 \%)$ & $1231(29.0 \%)$ & \\
\hline - $\quad 51-60$ & 175 (30.3\%) & $1325(36.1 \%)$ & $1500(35.3 \%)$ & \\
\hline$-\quad>60$ & $31(5.4 \%)$ & $292(7.9 \%)$ & $323(7.6 \%)$ & \\
\hline HCWs n. (\%) & $522(90.3 \%)$ & $3232(88.0 \%)$ & 3754 (88.3\%) & \multirow[t]{2}{*}{0.110} \\
\hline Non-HCWs n. (\%) & $56(9.7 \%)$ & $440(12.0 \%)$ & $496(11.7 \%)$ & \\
\hline Physicians n. (\%) & 78 (13.5\%) & 539 (14.7\%) & $617(14.5 \%)$ & \multirow{6}{*}{0.000} \\
\hline Nurses n. (\%) & $261(45.2 \%)$ & 1459 (39.7\%) & 1720 (40.5\%) & \\
\hline Nurses aids n. (\%) & $126(21.8 \%)$ & 619 (16.9\%) & 745 (17.5\%) & \\
\hline Others HCW n. (\%) & 57 (9.9\%) & 615 (16.7\%) & $672(15.8 \%)$ & \\
\hline Technicians n. (\%) & $20(3.5 \%)$ & $201(5.5 \%)$ & $221(5.2 \%)$ & \\
\hline Clerks n. (\%) & $36(6.2 \%)$ & $239(6.5 \%)$ & 275 (6.5\%) & \\
\hline \multicolumn{5}{|l|}{ Wards } \\
\hline - Geriatric I n. (\%) & 20 (46.51\%) & $23(53.49 \%)$ & $43(100 \%)$ & \multirow{13}{*}{0.000} \\
\hline - Infective diseases n. (\%) & $23(47.9 \%)$ & $25(52.1 \%)$ & $48(100 \%)$ & \\
\hline - Long-term Covid n. (\%) & $26(57.8 \%)$ & $19(42.2 \%)$ & $45(100 \%)$ & \\
\hline - Medicine I and II n. (\%) & $57(27.9 \%)$ & $147(72.1 \%)$ & $204(100 \%)$ & \\
\hline - Emergency medicine n. (\%) & $18(30.5 \%)$ & $41(69.5 \%)$ & $59(100 \%)$ & \\
\hline - Pulmonology n. (\%) & $26(21.85 \%)$ & $93(78.15 \%)$ & $119(100 \%)$ & \\
\hline - First aids n. (\%) & $51(18.75 \%)$ & $221(81.25 \%)$ & $272(100 \%)$ & \\
\hline - Rehabilitation n. (\%) & $22(22.2 \%)$ & $77(77.8 \%)$ & $99(100 \%)$ & \\
\hline - Others n. (\%) & $126(9.7 \%)$ & $1175(90.3 \%)$ & $1301(100 \%)$ & \\
\hline - Surgeries n. (\%) & $95(10.5 \%)$ & $814(89.5 \%)$ & $909(100 \%)$ & \\
\hline - Administration/technicians n. (\%) & $50(11.9 \%)$ & $371(88.1 \%)$ & $421(100 \%)$ & \\
\hline - Anesthesia and resuscitation n. (\%) & $17(8.6 \%)$ & $181(91.4 \%)$ & $198(100 \%)$ & \\
\hline - Other medicines n. (\%) & $26(7.3 \%)$ & $329(92.7 \%)$ & 355 (100\%) & \\
\hline
\end{tabular}

Page 11/15 


\begin{tabular}{|c|c|c|c|c|}
\hline - Radiology and nuclear medicine n. (\%) & $21(11.8 \%)$ & $157(88.2 \%)$ & $178(100 \%)$ & \\
\hline $\mathrm{N}^{\circ}$ PCR tests per person, median (IQR) & $14(10-19)$ & $10(7-10)$ & $10(7-14)$ & 0.03 \\
\hline Symptoms at the first PCR + n. (\%) & $301(52.1)$ & - & $301(52.1)$ & \\
\hline Symptoms recorded at the last PCR $+\mathrm{n} .(\%)$ & $483(83.6)$ & - & $483(83.6)$ & \\
\hline Asymptomatic n. (\%) & $95(16.4)$ & - & $95(16.4)$ & \\
\hline Upper respiratory symptoms n. (\%) & $315(54.5)$ & - & $315(54.5)$ & \\
\hline Fever $>37.5^{\circ} \mathrm{C}$ n. (\%) & $273(47.2)$ & - & $273(47.2)$ & \\
\hline Loss of taste and smell n. (\%) & $161(27.8)$ & - & $161(27.8)$ & \\
\hline Lower respiratory symptoms n. (\%) & $33(5.7)$ & - & $33(5.7)$ & \\
\hline Diarrhea n. (\%) & $35(6.0)$ & - & $35(6.0)$ & \\
\hline Others (myalgia, malaise, headache) & $249(43)$ & - & $249(43)$ & \\
\hline
\end{tabular}

Table 2 Incidence of COVID-19 infection in hospital workers in Trieste from March 1, 2020 to February $28,2021$.

\begin{tabular}{|lllll|}
\hline Month & $\begin{array}{l}\text { Surveillance time, person- } \\
\text { days }\end{array}$ & $\begin{array}{l}\text { COVID-19 } \\
\text { cases }\end{array}$ & $\begin{array}{l}\text { Incident rate per 100.000 person- } \\
\text { days }\end{array}$ & $\begin{array}{l}95 \% \\
\text { Cl }\end{array}$ \\
\hline March & 130696 & 95 & 72.69 & $64-79$ \\
\hline April & 123870 & 71 & 57.32 & $48-66$ \\
\hline May & 126077 & 15 & 11.90 & $6.8-19$ \\
\hline June & 121680 & 0 & 0.00 & - \\
\hline July & 125705 & 0 & 0.00 & - \\
\hline August & 125705 & 4 & 3.18 & 7.9 \\
\hline September & 121650 & & 6.58 & $3-12$ \\
\hline October & 125674 & 8 & 55.70 & $46-64$ \\
\hline November & 119910 & 70 & 97.57 & $93-99$ \\
\hline December & 120249 & 117 & 93.97 & $88-98$ \\
\hline January & 116157 & 113 & 61.12 & $52-70$ \\
\hline February & 103236 & 71 & 13.56 & $8-22$ \\
\hline
\end{tabular}

Table 3 Incidence risk ratio (IRR) and 95\% confidence intervals (CI) of factors associated with COVID-19 infection in hospitals' workers calculated using the Poisson regression adjusted for age and number of PCR test performed 


\begin{tabular}{|lll|}
\hline WORKERS' CHARACTERISTICS & Adjusted IRR (95\% Cl) & P \\
\hline Age & $0.99(0.98-0.99)$ & 0.012 \\
\hline Sex: & & \\
\hline Men & $0.94(0.80-1.1)$ & 0.422 \\
\hline Women & 1 & \\
\hline Work tasks: & & \\
\hline Physician & 1 & 0.153 \\
\hline Nurse & $1.18(0.94-1.49)$ & 0.203 \\
\hline Nurse aid & $1.18(0.91-1.52)$ & 0.036 \\
\hline Other HCW & $0.70(0.50-0.98)$ & 0.223 \\
\hline Technician & $0.72(0.43-1.22)$ & 0.428 \\
\hline Clerks & $1.18(0.79-1.76)$ & \\
\hline Wards: & & 0.000 \\
\hline Other Medicines & 1 & 0.751 \\
\hline Anesthesia and resuscitation & $1.10(0.61-1.97)$ & 0.060 \\
\hline Others & $1.48(0.98-2.23)$ & 0.000 \\
\hline Surgeries & $1.37(0.90-2.08)$ & 0.135 \\
\hline Radiology and nuclear medicine & $1.96(1.14-3.36)$ & 0.015 \\
\hline Administration and technicians & $1.85(1.13-3.04)$ & 0.014 \\
\hline First aid & $2.44(1.56-3.83)$ & 0.000 \\
\hline Pneumology - Covid & $2.72(1.65-4.48)$ & 0.000 \\
\hline Rehabilitation - Covid & $3.59(2.14-6.04)$ & 0.000 \\
\hline Internal medicine and clinical medicine - pre-Covid & $3.52(2.28-5.42)$ & 0.000 \\
\hline Emergency medicine & $3.70(2.15-6.37)$ & 0.000 \\
\hline Geriatry - Covid & $5.96(3.64-9.76)$ & 0.000 \\
\hline Infective diseases - Covid & $5.75(3.54-9.33)$ & \\
\hline Long term - Covid & $7.15 .59-11.14)$ & 0.000 \\
\hline
\end{tabular}

Table 4 Incidence of COVID-19 infection (95\% Confidence Intervals Cl) in hospital workers in Trieste from March 1, 2021 to May 31, 2021 in vaccinated and non-vaccinated workers. 


\begin{tabular}{|c|c|c|c|c|c|c|c|c|c|}
\hline \multirow[t]{2}{*}{ Month } & \multirow{2}{*}{$\begin{array}{l}\text { Surveillance } \\
\text { time, } \\
\text { person-days } \\
\text { Vaccinated }\end{array}$} & \multicolumn{2}{|c|}{$\begin{array}{l}\text { COVID-19 } \\
\text { cases } \\
\text { vaccinated }\end{array}$} & \multirow{2}{*}{$\begin{array}{l}\text { Surveillance } \\
\text { time, } \\
\text { person-days } \\
\text { Non- } \\
\text { vaccinated }\end{array}$} & \multicolumn{2}{|c|}{$\begin{array}{l}\text { COVID-19 } \\
\text { cases non- } \\
\text { vaccinated }\end{array}$} & \multicolumn{3}{|c|}{$\begin{array}{l}\text { Incidence rate ratio } \\
(95 \% \mathrm{Cl})\end{array}$} \\
\hline & & $\mathrm{n}$. & $\begin{array}{l}\text { Incident } \\
\text { rate per } \\
100.000 \\
\text { person- } \\
\text { days }\end{array}$ & & $\mathrm{n}$. & $\begin{array}{l}\text { Incident } \\
\text { rate per } \\
100.000 \\
\text { person- } \\
\text { days }\end{array}$ & Unadjusted & Adjusted* & $\begin{array}{l}\mathrm{p}- \\
\text { value }\end{array}$ \\
\hline March & 81964 & 4 & 4.88 & 31868 & 33 & 103.55 & $\begin{array}{l}0.05(0.02- \\
0.1)\end{array}$ & $\begin{array}{l}0.05 \\
(0.02- \\
0.08)\end{array}$ & $<0.001$ \\
\hline April & 79200 & 2 & 2.53 & 29850 & 15 & 50.25 & $\begin{array}{l}0.05(0.02- \\
0.1)\end{array}$ & $\begin{array}{l}0.05 \\
(0.02- \\
0.08)\end{array}$ & $<0.001$ \\
\hline May & 81778 & 2 & 2.45 & 30721 & 4 & 13.02 & $\begin{array}{l}0.19(0.16- \\
0.28)\end{array}$ & $\begin{array}{l}0.20 \\
(0.16- \\
0.30)\end{array}$ & $<0.01$ \\
\hline
\end{tabular}

*Using Poisson regression as reported in M\&M section

\section{Figures}
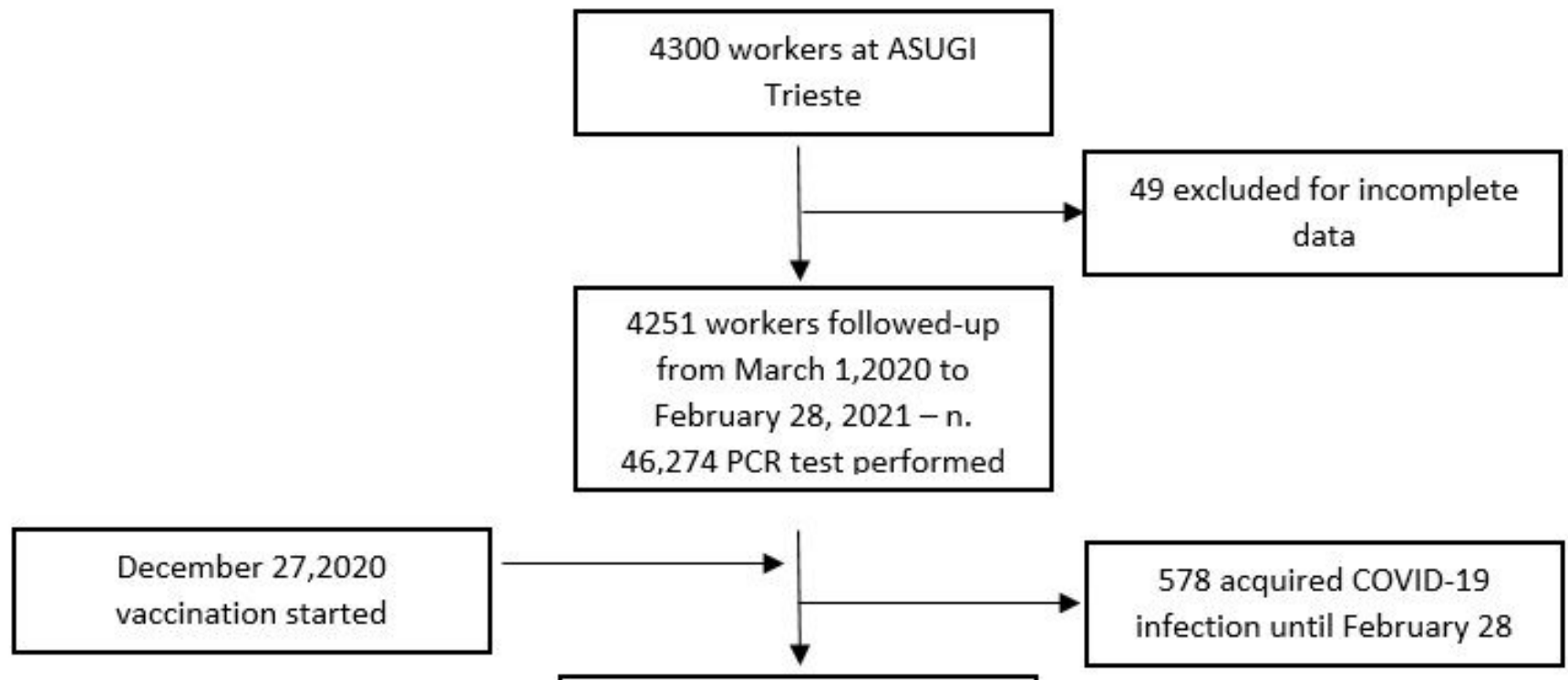

578 acquired COVID-19 infection until February 28

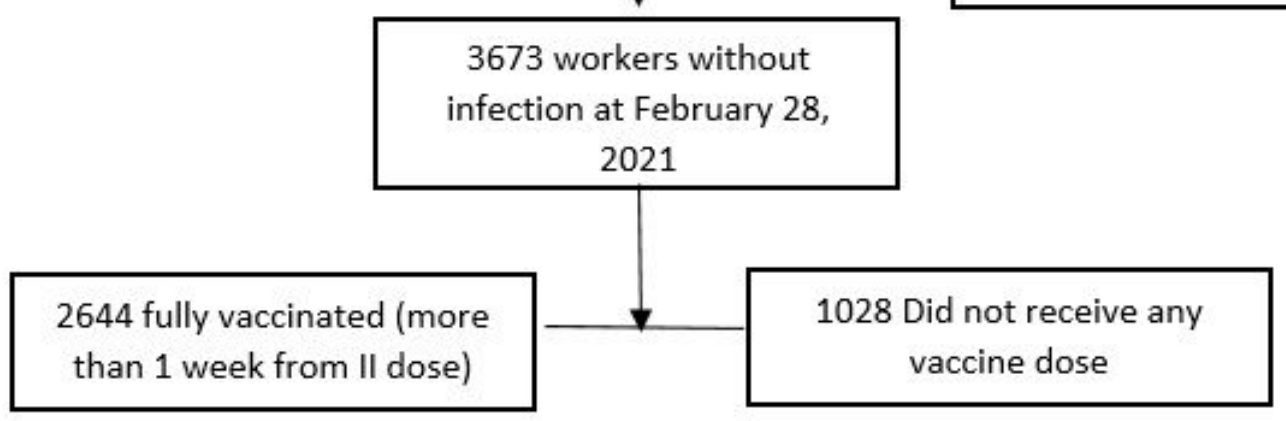

Figure 1 
Study cohort analyzed (PCR=polymerase chain reaction in nasopharyngeal swabs)

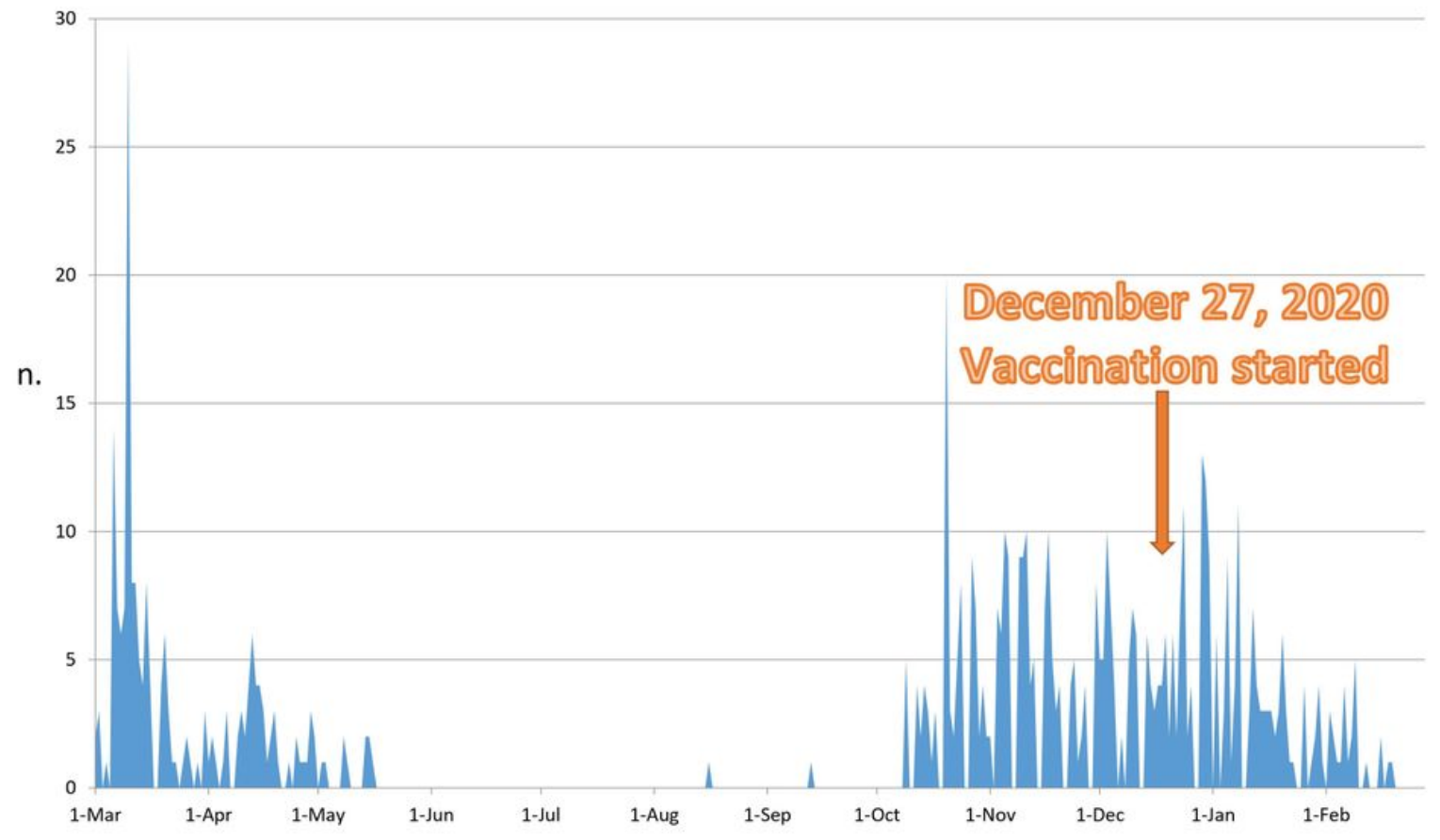

\section{Figure 2}

Incidence cases of COVID-19 in health care workers from March 1, 2020 to February 28, 2021 